\title{
Lexical Decision Based on Homograph's meaning Frequency and Working Memory
}

\author{
Peiran Wang \\ Department of Psychology, the University of Texas at Austin, Austin, 78712, USA \\ alywang7@gmail.com
}

Keywords: lexical ambiguity; homograph; context, working memory.

\begin{abstract}
Words that have more than one meaning are called homographs. Homographs create lexical ambiguity, where listeners have to determine which meaning of the word was intended. Past studies noted the importance of context and working memory on lexical decision. The congruency between context and another word presented afterwards generates faster response; higher working memory facilitates robust switch between different meanings, which leads to faster response also. Our research examined the effect of homograph's meaning frequency and working memory on lexical decision. However, we focus on the subordinate context as the basis for lexical response. Based on the past findings, we expected to see that the congruency between context and target word led to faster lexical response.
\end{abstract}

\section{Introduction}

A word that contains several meanings can create ambiguity in conversation and different factors help people select the appropriate meaning to interpret the sentences. The term "homograph" means a word that has more than one meaning [1]. For example, the word "bank" refers to a "financial institution", but also has the subordinate meaning referring to a "river bank". In most current studies, researchers investigated several factors that influence people's interpretation of the homographs: working memory, context, and age. Homographs create "lexical ambiguity", because readers or listeners have to determine which meaning of the word was intended [3].

Past research studies examined the effect of context and working memory on lexical decision making in essence. Context makes subjects to activate the contextually appropriate meaning, and the meaning may match or be conflict with the target word that is presented afterwards. The incongruence between the context and target leads to slower response whereas the congruency generates faster response [4]. Furthermore, researchers found out that higher working memory helps with lexical response because subjects could robustly switch between different meanings [2]. Our research mainly focuses on the effect of word's meaning frequency and working memory on lexical decision making. The meaning frequency is determined by the order of lexical entry in dictionary. The experiment primarily emphasizes how subordinate context influences lexical response by presenting sentences that biased toward subordinate meaning of homographs. First, I will discuss the influence of context on the interpretation of homographs. It is followed by the illustration of the working memory’s influence on lexical performance.

\section{Past Studies}

\section{1 context effects on lexical priming of homographs}

Context influences people's lexical decision significantly, according to past studies. In the prior experiments, a homograph and another word that was related to one of the homograph's meanings were shown in a pair; two words creates a context. After certain time a target word was presented and subjects needed to judge whether the target word was a real word. Researchers usually consider congruency as an aspect of the context: the relationship between the target word and the context. Congruence meant that the target word matched the intended meaning of the context (e.g. context: "bat", "baseball”, target word: "game”). In the contrast, incongruence occurred when the target did 
not match the intended meaning of the context (e.g. context: “clothes", "tag”, target word: "price”). There are also conditions where the target words were not related to the context meaning at all (context: "subject”, "school”, target word: “water"). Swinney and Hakes noted that the disambiguating cue in a context could help reduce the processing load as people activated the appropriate meaning of a homograph immediately after the presence of disambiguation [6].

Researcher concluded that dominant meaning of an ambiguity was activated faster in a neutral context than subordinate meaning; the constrained context would help speed the activation of the contextually appropriate meaning [5]. They found the dominant target was still activated slightly faster than the subordinate target in a subordinate constrained context [7]. However, the subordinate context speeded the lexical retrieving process in the subordinate target condition, and the reaction time was much less than when the subordinate target word paired up with a dominant context sentence [7].

\section{2 working memory effects}

Working memory has become a more popular area that researchers analyze regarding its effect on lexical decision. Gadsby, Arnott, and Copland [1] employed the methodology of Nievas and Beffa [4] to investigate the impact of working memory capacity on the inhibitory aspects of lexical ambiguity resolution. Participants were divided into three groups based on their working memory performance in a task. They were asked to listen to a list that had five, six, seven, or eight words total that had matched frequency, concreteness, and imageability [1]. At first, the participants listened to the list of words, memorized as many as they could, and finally wrote those down they remembered in the same order as they were on the list immediately. Those who recalled five words and more were placed in the high working memory group, and the rest were placed in low working memory group.

As a result, participants with low working memory showed less priming effects when the context was subordinate. This might be due to the insufficient resources left to support activation of related words after decent amount of resources used in the relatedness judgment [1]. The high working memory group illustrated activation for both dominant and subordinate meaning, and their priming effects were not affected by the relatedness judgment.

\section{Current Study}

In our own research, we explored how a homograph's meaning frequency and working memory influences people's lexical decision. Furthermore, the impact of inter stimulus interval (ISI) was also studied. Both the individual and combined effect of these factors were investigated. The purpose of the experiment was to test whether multiple meanings of a lexically ambiguous word are activated after that word appears in a sentence, and the time course of activation. Past studies did not look deep into the effect of frequency difference between the dominant meaning and subordinate meaning on lexical decision, which is defined by the order of lexical entry in the dictionary. In addition, subordinate context is emphasized in our study: subjects will see sentences that biases toward the subordinate meaning of homographs, interspersed by some control sentences that do not contain homographs. Target words are presented after the sentences, which are either real words or pseudo words. Participants need to judge whether the target words shown after certain time interval are actual words. The control sentences are added in order to prevent participants noticing the purpose of the study. There are three potential aspects I would like to investigate: 1.How congruency between context and target word influences lexical response 2.How working memory influences the lexical response. We hypothesized that people with higher working memory would activate different meanings more robustly than those with lower working memory. In addition, we believed that the congruency between sentences and words resulted in faster response.

The frequency is determined by the order of lexical entry in the dictionary. For example, the word "bat" has several meanings. The first meaning shown in the dictionary is "any of various types of club with a handle, used to hit the ball in certain sports, such as cricket, baseball, or table tennis", and the third meaning is "a nocturnal mouselike animal flying with a pair of membranous wings". We will use the first meaning as the dominant meaning and third meaning as the subordinate meaning. And 
we will present target words that correspond to the homographs' dominant meanings and subordinate meanings. In this case, we take all the first meanings of homographs as their dominant meaning as third meanings as subordinate meaning. Sentences that have subordinate context will be shown first, after certain time interval the target words will be presented and subjects will judge whether they relate to the sentence.

Also, we look at working memory as another potential factor that affects lexical decision. This experiment divides the words into four conditions: dominant condition, subordinate condition, unrelated condition, and pseudo word condition. The design replicated some of the past studies where the sentences create contexts; later target words will be shown. The experiment examined the effect of working memory on three conditions: subordinate context- dominant target, subordinate contextsubordinate target, and subordinate context- unrelated target. The control sentences were used as filter sentences; therefore, they were not included in data analysis.

\section{Methods}

\section{1 participants}

We recruited 36 college students who were native English speakers at different sites of UT campus for the study, and they were rewarded five dollars cash for their participation.

\section{2 materials}

California Verbal Learning Test (CVLT) was used to assess working memory because past studies also used it as a measuring tool for working memory. A list of 36 sentences that contained homographs and another set of 36 words were selected as the material presented to subjects. There were 12 words that related to the dominant meanings or subordinate meanings of the homographs, respectively; other 12 words that did not relate to the homograph's meaning at all. In addition, there were 36 control sentences that do not contain ambiguous words with pseudo words presented afterwards. The order of the sentences and words was counterbalanced; each subject was shown all the sentences and equal numbers of target words' conditions. The stimulus was programmed into software PsychoPy. Participants’ response times were recorded in the software.

\section{3 design}

The experiment used a $3 * 2 * 2$ design, with word type (dominant, subordinate, and unrelated) as a within subject variable, and ISI (0 vs. 300 ms) and Working Memory (high vs. low) as between subject variable.

\section{4 procedure}

Subjects completed the CVLT for working memory test first. They listened to an audio file that included two shopping lists A and B, and they were required to memorize items only from list A. They wrote down the items that were recalled on a sheet of paper. Based on the numbers of memorized words, they were divided to either high working memory group (greater or equal to 8) or low working memory group (less or equal to 7). They were seated in front of a computer with headphones on, and pairs of sentences that have subordinate context were shown on the screen first, and then the target words were presented after an ISI of 0/300 ms. Half of the subjects were assigned to $0 \mathrm{~ms}$ and another half was assigned to $300 \mathrm{~ms}$ as ISI. Then the subjects needed to decide whether the target words were real words by pressing " $y$ " as yes and " $n$ ” for no. The response time was recorded after each response is made. Table 1 is an example of a set of words shown on the screen:

Table 1 Words shown on the screen

\begin{tabular}{cc}
\hline Sentence (Subordinate/Control) & Target Word \\
\hline $\begin{array}{c}\text { Just before students need to be in their classrooms, the belling } \\
\text { will } \text { ring. }\end{array}$ & $\begin{array}{c}\text { Wedding (Dominant)/ Bell (Subordinate)/Bottle } \\
\text { (Unrelated) }\end{array}$ \\
$\begin{array}{c}\text { John bought ticket to attend a concert to see his favorite pop } \\
\text { star. }\end{array}$ & $\begin{array}{c}\text { Sky (Dominant)/Celebrity (Subordinate)/Soap } \\
\text { (Unrelated) } \\
\text { Oriday's test is cancelled as some students celebrate their } \\
\text { religious holiday. }\end{array}$ \\
$\begin{array}{c}\text { The potluck was a success: people enjoyed food from } \\
\text { different culture. }\end{array}$ & Overend (Pseudo word) \\
\hline
\end{tabular}


Subjects will do a set of practice trials to get familiar with the procedure, and those will be shown before the experimental trials. Also, the control trials that have pseudo words after sentences are designed to hide the true purpose behind the study. Most of the other studies used sentences that have both dominant and subordinate context; in the contrast, our study focuses primarily on the effect of subordinate context and lexical decision. Furthermore, we operationally define the meaning frequency with the order of the meaning in the dictionary.

\section{5 result}

We ran a repeated ANOVA with the condition as within-subject variable and both ISI and Working Memory as between-subject variable. As a result, we did not find any independent significance of the variables. However, there were two interactions nearly approached significance. The interaction between Condition and ISI $(\mathrm{P}=0.082, \mathrm{f}=2.595)$ and the interaction between Condition and Working Memory $(\mathrm{P}=0.084, \mathrm{f}=2.572)$ were almost significant. We believed these nearly significant results were due to the insufficient number of participants.

(1) Condition * ISI

The immediate presence of target words after sentences (ISI=0 ms) supported our hypothesis that congruency between sentence contexts and target words influence people's lexical performance. The delay of target words resulted in the activation of dominant meanings and loss of subordinate meanings. When the ISI was $300 \mathrm{~ms}$, participants responded fastest when target words were dominant $(\mathrm{M}=0.759 \mathrm{~s})$. Both the dominant condition and subordinate condition ( $\mathrm{M}=0.831 \mathrm{~s})$ resulted in faster response than unrelated condition ( $\mathrm{M}=1.079 \mathrm{~s})$. In the contrast, subordinate meanings of homographs were activated sooner $(M=0.574 \mathrm{~s})$ than were dominant meanings $(\mathrm{M}=0.752 \mathrm{~s})$ if the ISI was $0 \mathrm{~ms}$. There was an interesting finding: reaction time in unrelated condition $(\mathrm{M}=0.681 \mathrm{~s})$ was less than dominant condition when ISI=0 ms. In other words, the congruency between sentences and target words helped with response if target words were presented immediately after sentences. Nonetheless, the activation of subordinate meanings disappeared quickly and dominant meanings were primed after 300 ms. The sentence context did influence people's response when the ISI was 0 ms. Table 2 and Fig. 1 demonstrated this result.

Table 2 Condition*ISI

\begin{tabular}{|c|c|c|c|c|c|c|c|}
\hline \multicolumn{3}{|c|}{ Source } & $\begin{array}{c}\text { Type III Sum of } \\
\text { Squares }\end{array}$ & df & Mean Square & $\mathrm{F}$ & Sig. \\
\hline \multicolumn{3}{|r|}{ Sphericity Assumed } & 0.660 & 2 & 0.330 & 2.595 & 0.082 \\
\hline \multirow{3}{*}{\multicolumn{2}{|c|}{ Condition * ISI }} & Greenhouse-Geisser & 0.660 & 1.722 & 0.383 & 2.595 & 0.091 \\
\hline & & Huynh-Feldt & 0.660 & 1.982 & 0.333 & 2.595 & 0.083 \\
\hline & & Lower-bound & 0.660 & 1.000 & 0.660 & 2.595 & 0.117 \\
\hline \multirow{2}{*}{ ISI } & \multirow{2}{*}{ Condition } & \multirow{2}{*}{ Mean } & \multirow{2}{*}{ Std. Error } & \multicolumn{4}{|c|}{ 95\% Confidence Interval } \\
\hline & & & & \multicolumn{2}{|c|}{ Lower Bound } & \multicolumn{2}{|c|}{ Upper Bound } \\
\hline \multirow{3}{*}{ A } & 1 & 0.759 & 0.104 & \multicolumn{2}{|c|}{0.547} & \multicolumn{2}{|c|}{0.970} \\
\hline & 2 & 0.831 & 0.093 & \multicolumn{2}{|c|}{0.642} & \multicolumn{2}{|c|}{1.020} \\
\hline & 3 & 1.079 & 0.137 & \multicolumn{2}{|c|}{0.799} & \multicolumn{2}{|c|}{1.359} \\
\hline \multirow{3}{*}{ B } & 1 & 0.752 & 0.109 & \multicolumn{2}{|c|}{0.529} & \multicolumn{2}{|c|}{0.975} \\
\hline & 2 & 0.574 & 0.098 & \multicolumn{2}{|c|}{0.375} & \multicolumn{2}{|c|}{0.773} \\
\hline & 3 & 0.681 & 0.145 & \multicolumn{2}{|c|}{0.386} & \multicolumn{2}{|c|}{0.976} \\
\hline
\end{tabular}




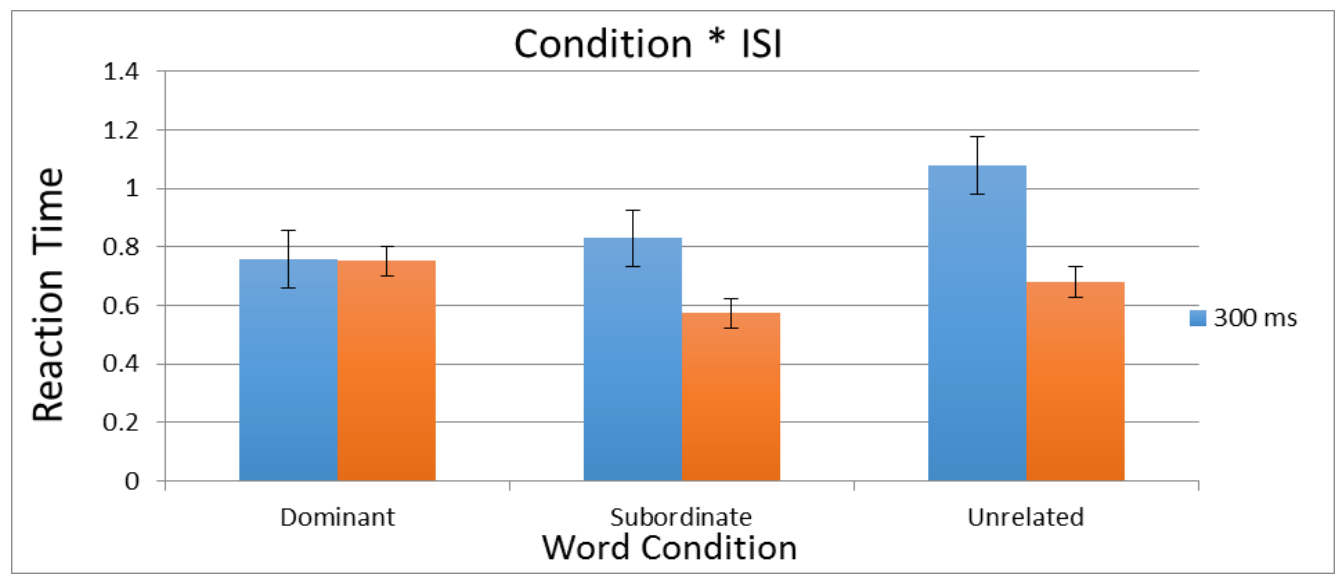

(2) Condition * Working Memory

Fig. 1 Condition * ISI

Table 3 Condition * WM

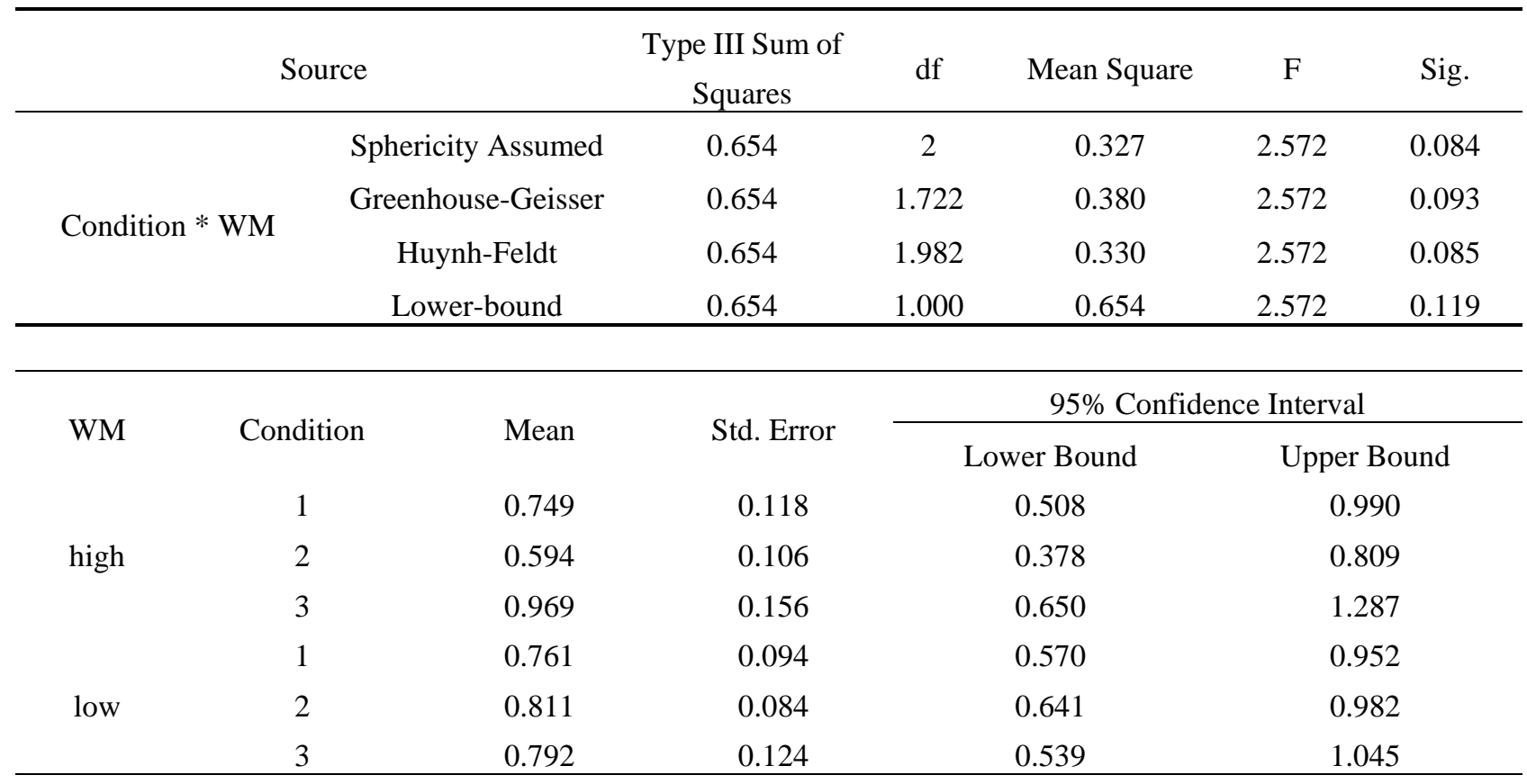

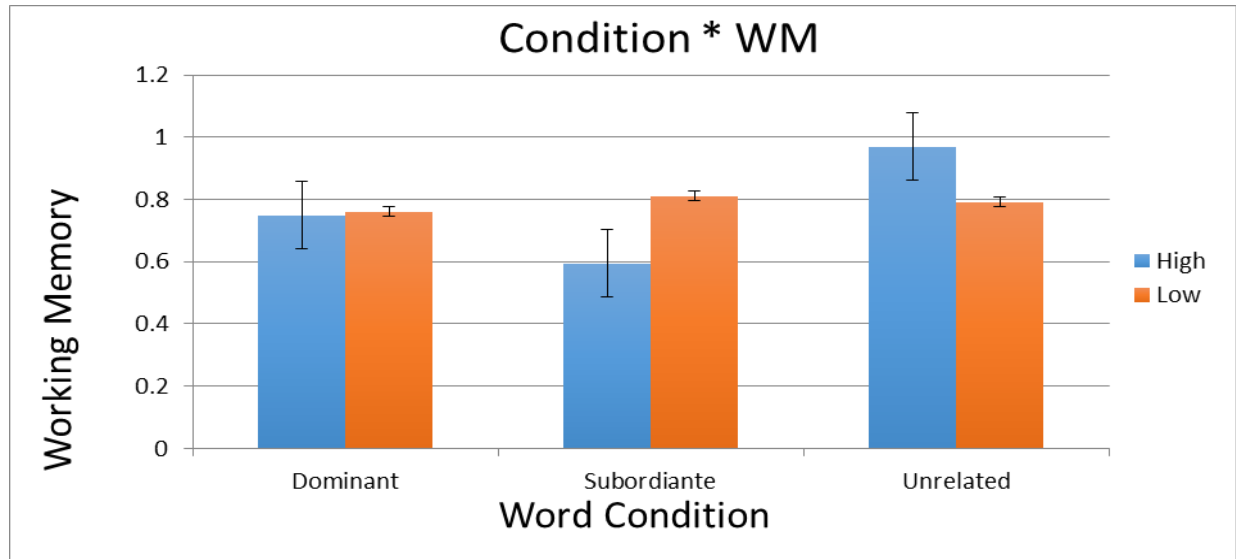

Fig. 2 Condition *WM

People with higher working memory were more robust with switching between homographs' different meanings. This supported Gunter, Wagner, and Friederici's conclusion. Also, the context did help with their response. Subordinate meanings were primed the fastest $(\mathrm{M}=0.594 \mathrm{~s})$, followed by dominant meaning $(\mathrm{M}=0.749 \mathrm{~s})$ and unrelated meanings $(\mathrm{M}=0.969 \mathrm{~s})$. This also supported our hypothesis. Conversely, people with lower working memory experienced trouble with activating contextually appropriate meanings. Dominant meanings were primed first through each trial 
( $M=0.761 \mathrm{~s})$, and they had difficulty activating subordinate meanings ( $M=0.811 \mathrm{~s})$. The sentence context did not affect their lexical response. Table 3 and Fig. 2 presented this finding.

\section{Conclusion}

In essence, majority of our hypothesis was corroborated by the data. Although there's no statistically significant effect, two interactions almost approached significance. It is possible that higher number of participants can result in significance. The congruency between sentence contexts and target words generated faster response among participants with higher working memory when the target words were shown immediately after sentences. The activation of subordinate meanings disappeared after $300 \mathrm{~ms}$ accompanied by the activation of dominant meanings. In addition, people with higher working memory were more skilled at switching between different meanings of homographs than those with lower working memory. For people with lower working memory, the dominant meanings were always primed first rather than contextually appropriate meanings.

\section{References}

[1] Gadsby, N., Arnott, W. L., \& Copland, D. A. (2008). An investigation of working memory influences on lexical ambiguity resolution. Neuropsychology, 22 (2), 209-216.

[2] Gunter, T. C., Wagner, S., \& Friederici, A. D. (2003). Working Memory and Lexical Ambiguity Resolution as Revealed by ERPs: A Difficult Case for Activation Theories. Journal of Cognitive Neuroscience, 15 (5), 643-657.

[3] Lee, C., \& Federmeier, K. D. (2011). Differential age effects on lexical ambiguity resolution mechanisms. Psychophysiology, 48 (7), 960-972.

[4] Nievas, F., \& Marí-Beffa, P. (2002). Negative priming from the non-selected meaning of the homograph. British Journal of Psychology, 93 (1), 47-66.

[5] Simpson, G.B., \& Burgess, C. (1985). Activation and solution processes in the recognition of ambiguous words. Journal of Experimental Psychology: Human Perception and Performance, 11, 28-39.

[6] Swinney, D. A., \& Hakes, D. T. (1976). Effects of prior context upon lexical access during sentence comprehension. Journal of Verbal Learning \& Verbal Behavior, 15 (6), 681-689.

[7] Tabossi, P., \& Zardon, F. (1993). Processing ambiguous words in context. Journal of Memory and Language, 32 (3), 359-372. 